\title{
Morphophysiological and molecular effects of drought stress in rice Shamsun Nahar ${ }^{1}$, Jyotirmay Kalita ${ }^{1}$, Lingaraj Sahoo ${ }^{2}$ and Bhaben Tanti ${ }^{1, *}$
}

${ }^{1}$ Department of Botany, Gauhati University, Guwahati-781014, Assam, India.

2Department of Biosciences and Bioengineering, Indian Institute of Technology Guwahati, Guwahati781039, Assam, India.

Received: July 24, 2016; Accepted: August 17, 2016

\begin{abstract}
Drought is a major abiotic stress that adversely affects the rice growth, mostly in the rainfed ecosystem that ultimately affects the biomass production and yield. Rice needs to adapt a series of physiological mechanisms with complicated regulatory network to fight and cope up with the unfavourable conditions due to drought stress. Morphological and physiological response in rice include inhibition of seed germination, slower growth rate, low root and shoot length, lower chlorophyll content, stomatal closure, lower rate of photosynthesis, yield reduction etc. Stress condition further results in development of response at the molecular level by the generation of reactive oxygen species (ROS) such as $\mathrm{O}_{2}{ }^{*}, \mathrm{H}_{2} \mathrm{O}_{2},{ }^{1} \mathrm{O}_{2}, \mathrm{OH}^{*}$ etc. which incites oxidative stress in the plants. Oxidative stress is overcome by the inherent capacity of plants to produce antioxidant species which may be enzymatic or non-enzymatic in nature. If however antioxidant defence mechanism cannot overpower the ROS generated, they cause oxidative damage to the plant tissues such as lipid peroxidation, protein oxidation, DNA damage, etc. resulting in cell death. Unlike other stresses, drought affects the physiology and biochemistry of the rice which adversely affects in the morphology and consequently delimits the yield of the plant. Therefore, understanding the morphological, biochemical and molecular mechanisms involved in rice against drought is utmost necessary for rice breeders to improve the rice for drought tolerant/resistance varieties for future green revolution. In this review, an attempt has been made to highlight the complex regulatory network involved in rice against drought with special emphasis on morphological, physiological and molecular mechanisms and to discuss the prospective and challenges for future plant breeders.
\end{abstract}

Key words: Drought stress; rice; morphophysiological effect; molecular effect; reactive oxygen species

\section{Introduction}

Drought is one of the major environmental stresses causing growth retardation and yield loss of plants. Of all the abiotic stresses which grossly reduce the crop productivity in general, drought is the most threatening one. In the last few years, efforts have been made to identify drought resistant traditional rice varieties and subsequently to develop drought tolerant rice through breeding approaches. But due to poor understanding of the morphophysiological and molecular basis of the growth and yield of rice in drought conditions, breeding efforts to improve drought tolerance rice have been hampered (Tuberosa and Salvi, 2006). From application point of view, it is crucially important to understand the physiological and molecular effects of drought stress in rice and their expression in morphology of the plant. Therefore, in this review, an attempt has been made to explain the morphophysiological and molecular effects on rice in water limited condition.

Water is a part of several important biochemical reactions going on in plants and is very important for maintenance of healthy metabolism in them, and thus its scarcity leads to several complications at morphological and physiological level. The drastic changes in earth's climate resulting from global warming have changed rainfall patterns across the globe in recent times (Trenberth, 2011). Paddy rice which prefers submerged conditions for growth is thus severely affected by the lack of rainfall in places where it is cultivated. In a country like India where many regions lack basic irrigation facilities, severe drought can result in the loss of yield of important crops including rice. It is thus very important to understand the mechanisms controlling drought stress manifestation in rice plants and the inherent capacity of plants to tolerate or succumb to drought. In rice, drought stress leads to various changes in the plant morphology, physiology and biochemistry.

The healthy growth of rice depends on the results of collective effort of several environmental factors that affect its life. Plant responses to drought stress are very complex as stress itself involves various climatic, soil and agronomic factors, frequently complicated by substantial variation in timing of occurrence, duration and intensity. The general complexity of drought is often aggravated under rainfed conditions in marginal areas by erratic and unpredictable rainfall, and by the occurrence of high temperatures, high levels of solar radiation, and poor soil characteristics. The combined effect of one or more such stress alongwith drought often leads to a much complex response in the plants' metabolism, highly altered from those of the individual stresses acting alone (Mittler, 2006). This high variability in the nature of drought stress, aggravated by an insufficient understanding of its complexity, has made it difficult to identify specific

\section{${ }^{*}$ Corresponding Author:}

Dr. Bhaben Tanti, Ph.D.

Associate Professor,

Department of Botany, Gauhati University,

Guwahati 781014, Assam, India.

E-mail: btanti@gauhati.ac.in; bt53@,rediffmail.com

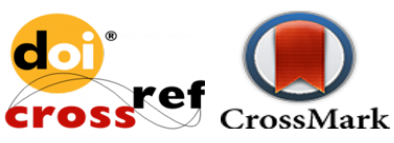


physiological traits required for improved crop performance under drought, consequently limiting plant breeding efforts to enhance crop drought tolerance. It is highly probable that optimal drought-adaptation requires the combination of several morphological, physiological, and phenological processes, which depend on a multitude of genes and varies within each target environment.

In addition to its direct effects on yield, drought can also reduce the potential beneficial effects of improved crop management practices such as fertilizer application or pest and disease management. Given the increasing scarcity of water resources, and competition for them, irrigation is not a practical option for alleviating drought in most of the rainfed areas. Drought management strategies therefore need to focus on maximizing extraction of available soil moisture and the efficiency of its use in crop establishment, growth, biomass and grain yield. However, agronomic and genetic options that do not involve external inputs of irrigation can only partially alleviate drought effects, because yield will always be lower than what can be achieved with irrigation.

This paper will discuss, from a physiological and breeding perspective, some of the current challenges and opportunities in genetic enhancement of drought resistance in rice and introduce some of the screening tools and methods.

\section{Morphological and physiological effects of drought on rice}

Drought stress leads to stomatal closure leading to limitation of gaseous exchange. The closure of stomata is controlled by phytohormones such as abscisic acid, cytokinnins, etc. (Daszkowska-Golec and Szarejko, 2013). Reduction of water content reduces stomatal activity and cell growth. Leaf area, cell size and intercellular volume decreases, and leaf rolling and death of leaf results from drought stress. In roots, drought results in reduction of meristematic activity, arresting root elongation. Suberization of the root system also results from water stress (Singh et al., 2012). Drought stress decreases the rate of photosynthesis by impairing pigments, photosystems, gas exchange, and key photosynthetic enzymes, thus affecting various steps in the photosynthetic pathway (Asharf and Harris, 2013), and this can ultimately reduce plant biomass and yield.

A comparative study of a drought tolerant (IRAT109) and a susceptible cultivar (Zhenshan97B) showed prominent variations of morphophysiological adaptations to drought conditions in both. Leaf rolling, reduction in plant height and grain yield was more pronounced in the susceptible cultivar. Leaves and stem showed better elongation in the tolerant cultivar. Drought resulted in increased panicle length in the tolerant cultivar while reduced panicle length in the susceptible one. Root length increase occurs in both cultivars; however, the increase is much more in the tolerant cultivar, showing this to be an important adaptive feature in drought tolerance. Photosynthetic rate of flag leaf increases more prominently in the tolerant cultivar. The tolerant cultivar also shows intense stomatal closure by reducing stomatal conductance more effectively than the susceptible cultivar (Ji et al., 2012). From an agronomic point of view, important component traits to be selected for drought resistance combined with decent yield are relative water content, panicle length, biomass yield, grains pre panicle, harvest index, lower leaf rolling, root/shoot length ratio, root length and drought recovery rate (Manickavelu et al., 2006). High leaf water potential is an important trait for drought resistance, minimizing the effects of drought on spikelet sterility and thus, on grain yields (Jongdee et al., 2002). Drought stress during panicle emergence prevents peduncle elongation, obstructing exertion of spikelet and causing sterility. Relative water content decreases and abscissic acid (ABA) content increases during drought, which also results in down-regulation of gibberelic acid (GA) biosynthesis genes. The ABA-GA antagonism is supposed to play a role in the failure of panicle exertions during drought (Muthurajan et al., 2011).

Silicon minimizes the effect of drought by enhancing the basal quantum yield, maximum quantum efficiency of PSII photochemistry, photosynthetic rate and transpiration rate of rice plants subjected to drought stress. The result is an enhanced dry matter accumulation, improved root system, leaf water content and chlorophyll content, while mineral content of leaves which increases under drought is brought down to the level of well watered plants (Chen et al., 2011). Moreover, microbial colonization of roots has proven to ameliorate the effects of drought stress on rice plants. Arbuscular mycorrhizal (AM) association with rice enhances photosynthesis and leads to increase in biomass, while also reducing the extent of oxidative injury (Ruíz-Sánchez et al., 2010). A consortium of Arospirillum brasilense and AM fungus Glomus intraradices show increased rice growth and photosynthesis, and increased antioxidant levels, while reducing lipid peroxidation under drought condition compared to the control (un-inoculated) plants or those inoculated with a single microbe. The co inoculation of $A$. brasilense leads to better colonization of rice roots by AM fungi (RuízSánchez et al., 2011). Trichoderma harzianum isolates resistant to dessication have also been shown to be able to induce drought tolerance in rice plants subjected to upto 9 days of drought treatment. $T$. harzianum colonized rice delayed wilting and showed promising growth with minimum oxidative stress (Shukla et al., 2012). Consortia of plant growth promoting rhizobcteria (PGPR) comprising strains 
of Pseudomonas, Arthrobacter, etc. enhance rice plant growth under drought and provide resistance to injury by activating the antioxidant defence mechanism of the plants (Shukla et al., 2012). Thus drought physiology of rice seems to be affected by external factors which could be a promising prospect in averting the harmful effects of drought. Here, Fig. 1 explains the effect of drought on morphology and physiology of rice.

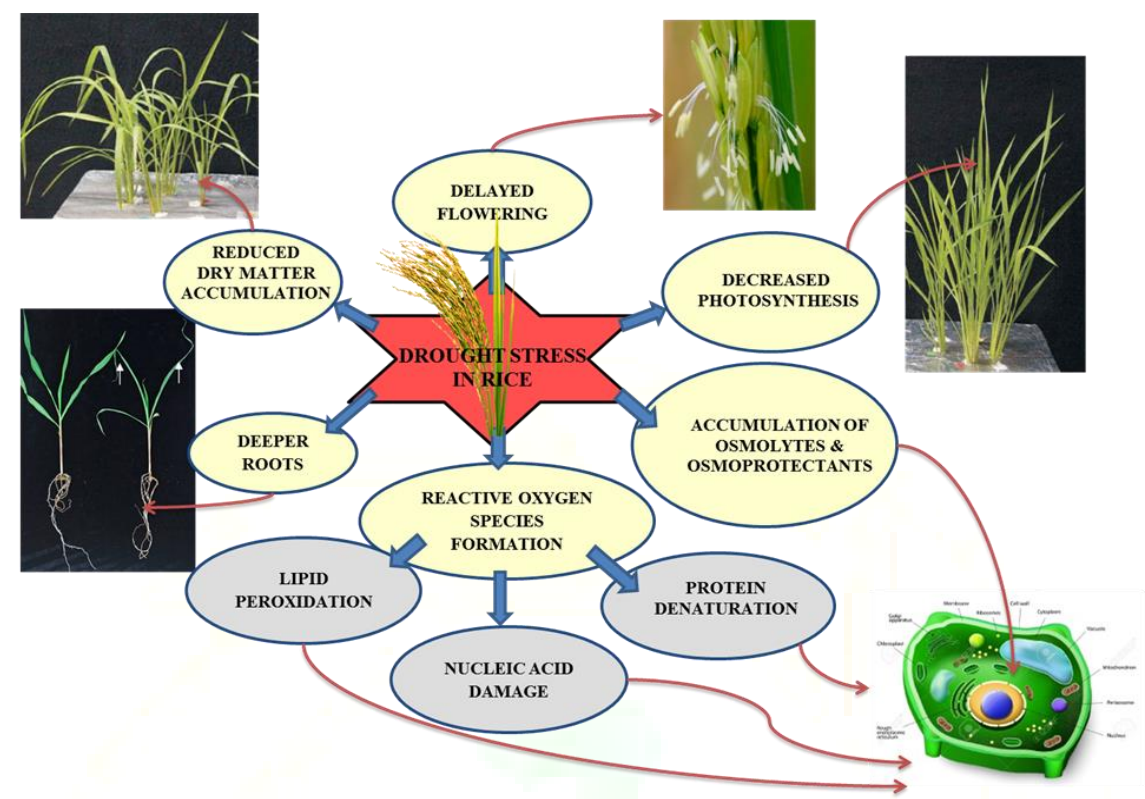

Figure 1: A schematic diagram showing morphological and physiological effect of drought in rice

\section{Biochemical effect of drought in rice}

At a molecular level, drought stress leads to the production of reactive oxygen species (ROS), leading to oxidative stress. Drought has been found to increase the level of superoxide radical $\left(\mathrm{O}_{2}-\right)$ and lipid peroxidation (LPO) as determined in terms of thiobarbituric acid (TBA) reactive substances (TBARS) in rice plants, while decreasing the concentration of total soluble proteins and thiols. Moreover, activities of antioxidant enzymes like superoxide dismutase (SOD), ascorbate peroxidase (APX), guaicol peroxidase (GPX), monodehydroascorbate reductase (MDHAR), dehydroascorbate reductase (DHAR), glutathione reductase (GR) have been found to increase in drought stressed rice plants (Sharma and Dubey, 2005). This shows the incitation of oxidative stress by drought and subsequent activation of the antioxidant defense mechanism in the rice plants (Fig. 2).

Drought tolerant varieties generally show better antioxidant production than susceptible ones (Lum et al., 2014). Increase in production of proline and soluble sugars have also been recorded under drought stress, with the increase more pronounced in tolerant varieties (Mostajeran and Rahimi-Eichi, 2009; Chutia et al., 2012). While proline has long been considered as an antioxidant, besides being an osmoprotectant, soluble sugars also act as osmoprotectants and signal in metabolic regulation.

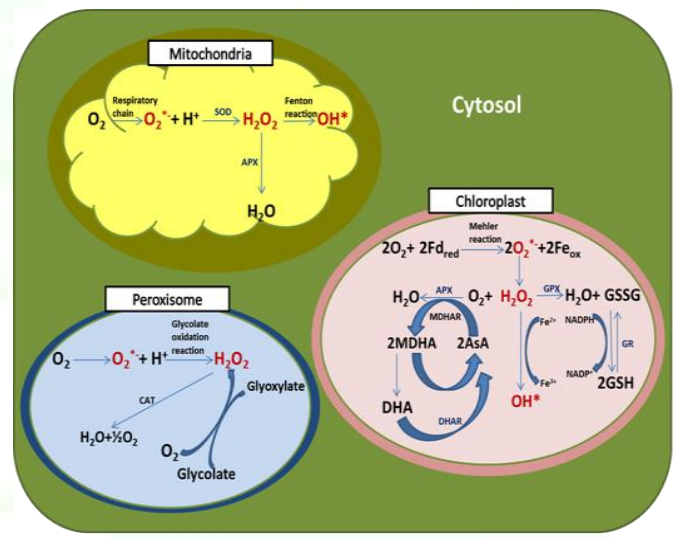

Figure 2: Schematic diagram showing production and scavenging of reactive oxygen species (ROS) during oxidative stress in rice at cellular level.

\section{Molecular effect of drought in rice}

Genes exclusively expressed in drought tolerant varieties include genes involved in signaling pathways, such as $\mathrm{Ca}^{2+}$ dependent protein kinase (CDPK) and ethylene responsive factors, genes involved in alteration of metabolism due to lower intracellular $\mathrm{CO}_{2}$ during drought, genes involved in decreasing oxidative injury, and genes maintaining cell turgor Rabello et al., 2008). A proteomic analysis of leaves of a drought avoiding upland (CT9993) and drought tolerant lowland (IR62260) rice variety during drought and on re-watering revealed the differential expression of 42 drought response proteins during drought, however, the changes in protein abundance being fully or substantially restored on re-watering. Identified among these 
proteins are RNA binding proteins and EF-Tu factor involved in protein synthesis, two isoforms of Rubisco activase, two isoforms of chloroplast Rieske FeS protein, chloroplastic fructose 1,6biphosphate aldolase, and cytosolic triose phosphate isomerase involved in photosynthesis and carbon metabolism, cytosolic and chloroplastic $\mathrm{Cu}-\mathrm{Zn} \mathrm{SOD}$, glutathione dihydroascorbate reductase and isoflavone reductase like protein, involved in cellular defense against oxidative injury during abiotic stress, nucleoside diphosphate kinase, involved in maintaining cellular levels of CTP, GTP and UTP, and actin depolymerizing factor, which might be involved in remodeling of the cytoskeleton to changes in the cell caused by water loss (Salekdeh et al., 2002). Xiong et al., (2010), found proteins peroxiredoxin and putative thioredoxin peroxidase involved in redox metabolism, sub units of $\mathrm{RuBisCO}$ involvd in photosynthesis, a putative actin binding protein involved in cytoskeleton remodeling, a putative chitinase involved in defense against pathogens, ribonuclease involved in protein metabolism, and voltage dependent anion selective channel protein and osmotin-like protein involved in signal transduction (Xiong et al., 2010). Another proteomic study revealed that Sadenosylmethionine (SAM) synthetase and xyloglucan endotransglycosylase (XET) are down regulated during drought, while actin-binding proteins (ADFs) and late embryogenesis abundant (LEA) proteins are up regulated (Chen et al., 2011). Down regulation of SAM synthetase and XET suggests disruption of cellular growth. ADFs play a role in adjusting the cellular actin cytoskeleton to change in cellular volume due to water loss and also stomatal regulation during water stress. LEA proteins are known to be protectants of membranes during desiccation (Tolleter et al., 2010) [Table 1].

Methionine (met) of proteins is a prime target of reactive oxygen species which oxidize it to methionine sulfoxide $(\mathrm{Met} O)$ during stress conditions in plants. The methionine sulfoxide reductase (MSR) proteins coded by 'Msr' genes in plants play important role in alleviating abiotic stresses by reducing $\mathrm{MetO}$ to Met state. CaMsrB2 gene from Capsicum annum, when expressed in transgenic rice has been found to improve tolerance to drought stress in terms of $\mathrm{H}_{2} \mathrm{O}_{2}$ production, leaf pigmentation, stomatal conductance, dark adapted quantum yield, osmotic potential, and plant and leaf temperature (Siddiqui et al., 2014).

Many genes which are differentially expressed during drought stress are spatially and temporally regulated. Photosynthesis-related genes are generally differentially expressed in leaf tissues, while genes involved in cell membrane formation and cell wall modification in root and young panicle. Similarly, expressions of genes vary at different growth stages such as tillering, panicle elongation, booting, etc.
Moreover, several genes such as NAC-domain protein 5-8, LEA protein, etc. are reciprocally expressed in different tissues during the same growth stage (Wang et al., 2011).

Apart from functional proteins like water stress inducible proteins (WSI76 and WSI724), dehydration inducible proteins (RD and ERD), etc., regulatory proteins such as basic region/Leu zipper motif (bZIP), protein kinases, etc. are important genes expressed in response to drought stress (Rabbani et al., 2003). Overexpression of the OsbZIP23 factor has been found to increase drought tolerance and improve yield in the rice crop under drought stress (Dey et al., 2016).

Table 1: Differently expressed proteins during drought stress in rice which subsequently alter different metabolic functions leading to a wide range of responses to the stress

\begin{tabular}{|c|c|}
\hline $\begin{array}{c}\text { Differently expressed proteins in } \\
\text { drought }\end{array}$ & Metabolic function \\
\hline RNA binding proteins & \\
\hline Ef-Tu factor & Protein synthesis \\
\hline Ribonuclease & \\
\hline Rubisco activase & \\
\hline Chloroplast Rieske FeS protein & \\
\hline $\begin{array}{l}\text { Chloroplastic fructose } \\
\text { biphosphate aldolase }\end{array}$ & Photosynthesis and carbon \\
\hline $\begin{array}{l}\text { Cytosolic triose phosphate } \\
\text { isomerase }\end{array}$ & \\
\hline RuBisCO & \\
\hline $\mathrm{Cu}-\mathrm{Zn}$ Superoxide dismutase $(\mathrm{Cu}-$ & \\
\hline Zn SOD) & \\
\hline Ascorbate peroxidase (APX) & \\
\hline Guaicol peroxidase (GPX) & \\
\hline $\begin{array}{l}\text { Monodehydroascorbate reductase } \\
\text { (MDHAR) }\end{array}$ & \\
\hline $\begin{array}{l}\text { Dehydroascorbate } \\
\text { (DHAR) }\end{array}$ & $\begin{array}{l}\text { Defense against oxidative } \\
\text { injury }\end{array}$ \\
\hline Glutathione reductase (GR) & \\
\hline $\begin{array}{l}\text { Glutathione dihydroascorbate } \\
\text { reductase }\end{array}$ & \\
\hline Isoflavone reductase & \\
\hline Peroxiredoxin & \\
\hline Thioredoxin peroxidise & \\
\hline $\begin{array}{l}\text { Voltage dependent anion selective } \\
\text { channel protein }\end{array}$ & Signal transduction \\
\hline Osmotin-like protein & \\
\hline Actin depolymerizing factor & Cytoskeleton and cellular \\
\hline Actin binding proteins & structure \\
\hline S-adenosylmethionine & \\
\hline Synthetase & Cellular growth \\
\hline Xyloglucan Endotransglycosylase & \\
\hline $\begin{array}{l}\text { Late Embryogenesis Abundant } \\
\text { (LEA) proteins }\end{array}$ & $\begin{array}{l}\text { Protection of membranes } \\
\text { against desiccation }\end{array}$ \\
\hline Chitinase & Defense against pathogens \\
\hline Nucleoside diphosphate kinase & $\begin{array}{l}\text { Cellular levels of CTP, GTP } \\
\text { and UTP }\end{array}$ \\
\hline
\end{tabular}

NAC transcription factors also appear to play a prominent role in stress tolerance mechanisms of rice and various other plants. NAC factors are responsible for development of the plant; however, stress responsive NAC (SNAC) genes appear to fall under a distinct group. SNACs are divided into three subgroups, SNAC-A, SNAC-B, and SNAC-C. Rice SNAC genes fall under the SNAC-A, and SNAC-B subgroups, while the SNAC-C subgroup is found to contain genes from lower groups of plants. The mode of functioning of SNAC genes 
during stress is by regulating biosynthesis of soluble sugars, level of free proline, antioxidants, $\mathrm{Na}^{+}$ accumulation, etc. The SNACs upregulate the expression of stress inducible genes such as OsLEA3. The stress tolerance mechanism of different SNAC genes however appear to be a complex one as their expression appear to be differently regulated and the growth phenotypes of different plants overexpressing different NAC genes are different (Nakashima et al., 2012).

Overexpression of OsRDCP1, which is involved in ubiquitination reaction, is found to increase drought tolerance in rice, suggesting that it might activate water stress related proteins by degradation of certain repressor proteins that block the drought stress response pathway (Bae et al., 2011). The DREB transcription factors are another important group of regulators that improve drought tolerance in rice. OsDREB2A overexpression using 4ABRC promoters has led to the development of drought tolerance in japonica variety TNG67 (Cui et al., 2011).

\section{Prospects for increasing drought tolerance in rice}

Rice comprises of 23 species under the genome Oryza, and 9 recognized types of genome. Such variations mean the presence of a large gene pool in nature, which can be screened for the presence of drought resistance traits for improvement of the crop. The genome type AA has been considered as the primary gene pool, genomes $\mathrm{BB}$ to $\mathrm{EE}$ as secondary gene pool and the rest as tertiary gene pool based on their phylogenetic relationships (Ge et al., 1999). These gene pools, particularly the tertiary gene pool, have been speculated to be able to contribute important trait genes to the rice cultivars. Cultivars having deep and thick roots are considered good for tolerating drought stress, while an erect flag leaf that enables photosynthesis for longer duration of times is also considered a positive trait for drought tolerance. Moreover, characters like early maturity, early vigor, rapid growth, diffusive resistance of stomata; leaf water retention, etc. are also considered beneficial for drought tolerance (Singh et al., 2012). There is a wide range of variation among rice cultivars cultivated throughout the world. Moreover, a wide range of wild varieties of the crop exist in nature. Transcriptome analysis of the wild rice Oryza rufipogon has led to the identification of 37 drought response genes differentially expressed in roots on application of drought stress (Tian et al., 2015). A transcription factor HYR which is associated with photosynthetic carbon metabolism has been identified, and its expression in rice has led to enhanced photosynthesis under drought and other environmental stresses, and resulted in increased yield under stress (Ambavaram et al., 2014).

Current technologies like use of functional molecular markers (FMM) derived from simple sequence repeats (SSR), single nucleotide polymorphism (SNP) etc. are very useful tools of crop improvement. Approaches like metabolomics, proteomics, association mapping etc. can be used for the identification of candidate genes. Such candidate genes can be used for the development of FMMs (Kage et al., 2016). SSRs are a type of variable number of tandem repeats (VNTR) which may be 2-6 base pair long, and are often codominant in nature. SSRs display a range of polymorphisms across related species, which can be harnessed for identification of genomic diversity of the crop. SNPs arise due to change in nucleotide sequence at a single base pair. These leads to the generation of allelic variation and development of new traits. Use of SSR mapping along with RFLP and AFLP has led to identification of QTLs responsible for root related traits of drought resistance in rice which also have pleiotropic effects on rice yield (Chandra et al., 2003). Marker assisted selection (MAS) has been used to improve root characteristics of IR64 and Kalinga-III, an upland rice variety, using QTLs from 'Azucena', a japonica variety. The selected plants with improved root characters were found to yield more under water stressed conditions (Tuberosa and Salvi, 2006). Whole genome resequencing has led to the detection of non-synonymous SNPs (nsSNPs) in 15 maize inbred lines with varied response to drought stress which has been used to identify 271 candidate genes for drought stress (Xu et al., 2014). Similar approaches in rice can be used to identify candidate genes responsible for drought tolerance, subsequently to be used in crop improvement programmes. SSR markers have been used for determining genetic diversity of rice, and establishing an association mapping panel out of a diverse collection of its cultivars (Nachimuthu et al., 2015). This is an important preliminary step before identification of candidate genes related to particular phenotypes. Such diversity panels have also been established for rice using SNP markers (Travis et al., 2015). Use of insertion and deletions (INDELs) as well as SNPs to study the diversity between drought susceptible and tolerant rice indicates that QTLs related to photosynthesis are particularly important in drought stress tolerance in rice (Zhang et al., 2016). The use of SSRs to determine diversity of drought responding Expressed Sequence Tags (ESTs) between upland and lowland rice cultivars, has led to further establishment of the correlation between uplandlowland differentiation and drought resistance, suggesting upland rice cultivars to be more tolerant to drought (Xia et al., 2014). Recently, candidate gene based SSR (cgSSR) markers have identified in rice with respect to salt stress response. Such novel markers bear great promise for diversity analysis of varieties differing in stress response (Molla et al., 2015). Identification of cgSSR markers relation to drought stress can be a way of tackling the problem of identifying important drought response genes. 
Such markers can also be developed using wild rice varieties showing drought tolerance, and can be a great boost in enriching the germplasm of the crop.

\section{Conclusion}

Understanding of genomic responses of drought stressed rice is of utmost importance as it determines the morphological and physiological changes due to the stress. In drought stress, most physiological and metabolic processes are affected by water deficits which ultimately affect the cell growth, stomatal regulation, photosynthesis, translocation, etc. For adaptation in drought stress, a large number of genes are regulated. For proper identification and exploitation of drought regulated genes in future crop improvement, analyses of large genetic populations is required. Moreover, it is difficult to select the component traits for grain yield of rice under drought stress, which also present challenges for dealing with a large number of genes even when identified in rice breeding programme.

A number of metabolic pathways are affected by drought, among which most prominent are photosynthesis and carbon metabolism and antioxidant defense mechanism which play significant roles under drought stress in rice. Therefore, emphasis should be given to the responsible genes of the above mentioned metabolic pathways for the development of drought resistant rice. Moreover, the effect of drought can be well reduced by a holistic approach involving selection for better component traits for yield, and better metabolism under drought, alongwith management of environmental conditions, such as soil silicon component as well as the rhizosphere microbial population.

\section{Acknowledgement}

The fellowship received from UGC - Maulana Azad National Fellowship for Minority Students, Govt. of India is highly acknowledged.

\section{References}

1. Ambavaram, M.M.R., Basu, S., Krishnan, A., Ramegowda, V., Batlang, U., Rahman, L., Baisakh, N., Pereira, A., Coordinated regulation of photosynthesis in rice increases yield and tolerance to environmental stress. Nature Communications. (2014): Doi: 10.1038/ncomms6302.

2. Asharf, M., Harris, P.J.C., Photosynthesis under stressful environments: An overview. Photosynthetica. 51.2, (2013): 163-190.

3. Bae, H., Kima, S.K., Cho, S.K., Kang, B.G., Kim, W.T., Overexpression of OsRDCP1, a rice RING domain-containing E3 ubiquitin ligase, increased tolerance to drought stress in rice (Oryza sativa L.). Plant Science. 180, (2011): 775-782.
4. Chandra, B.R., Nguyen, B.D., Chamarerk, V., Shanmugasundaram, P., Chezhian, P., Jeyaprakash, P., Ganesh, S.K., Palchamy, A., Sadasivam, S., Sarkarung, S., Wade, L.J., Nguyen, H.T., Genetic Analysis of Drought Resistance in Rice by Molecular Markers: Association between Secondary Traits and Field Performance. Crop Science. 43, (2003): 14571469.

5. Chen, W., Yao, X., Cai, K., Chen, J., Silicon Alleviates Drought Stress of Rice Plants by Improving Plant Water Status, Photosynthesis and Mineral Nutrient Absorption. Biol Trace Elem Res. 142, (2011): 67-76.

6. Chutia, J., Borah, S.P., Tanti, B., Effect of drought stress on protein and proline metabolism in seven traditional rice (Oryza sativa Linn.) genotypes of Assam, India. Journal of Research in Biology. 2.3, (2012): 206-214.

7. Cui, M., Zhang, W., Zhang, Q., Xu, Z., Zhu, Z., Duan, F., Wu, R., Induced over-expression of the transcription factor OsDREB2A improves drought tolerance in rice. Plant Physiology and Biochemistry. 49, (2011): 1384-1391.

8. Daszkowska-Golec, A., Szarejk,o I., Open or close the gate - stomata action under the control of phytohormones in drought stress conditions. Plant cell biology. 4, (2013): 1-16.

9. Dey, A., Samanta, M.K., Gayen, S., Sen, S.K., Maiti, M.K., Enhanced Gene Expression Rather than Natural Polymorphism in Coding Sequence of the OsbZIP23 Determines Drought Tolerance and Yield Improvement in Rice Genotypes. PLoS ONE. 11.3, (2016). Doi: 10.1371/journal.pone.0150763.

10. Ge, S., Sang, T., Lu, B.R., Hong, D.Y., Phylogeny of rice genomes with emphasis on origins of allotetraploid species. PNAS. 96.25, (1999): 1440014405.

11. Gusain, Y.S., Singh, U.S., Sharma, A.K., Bacterial mediated amelioration of drought stress in drought tolerant and susceptible cultivars of rice (Oryza sativa L.). African Journal of Biotechnology. 14.9, (2015): 764-773.

12. Ji, K., Wanga, Y., Sun, W., Lou, Q., Mei, H., Shen, S., Chen, H., Drought-responsive mechanisms in rice genotypes with contrasting drought tolerance during reproductive stage. Journal of Plant Physiology. 169, (2012): 336- 344.

13. Jongdee, B., Fukai, S., Cooper, M., Leaf water potential and osmotic adjustment as physiological traits to improve drought tolerance in rice. Field Crops Research. 76, (2002): 153-163.

14. Kage, U., Kumar, A., Dhokane, D., Karre, S., Kushalappa, A.C., Functional molecular markers for crop improvement. Critical Reviews in Biotechnology. 36.5, (2016): 917-930.

15. Lum, M.S., Hanafi, M.M., Rafii, Y.M., Akmar, A.S.N., Effect of drought stress on growth, proline 
and antioxidant enzyme activities of upland rice. The Journal of Animal \& Plant Sciences. 24.5, (2014): 1487-1493.

16. Manickavelu, A., Nadarajan, N., Ganesh, S.K., Gnanamalar, R.P., Chandra, B.R., Drought tolerance in rice: morphological and molecular genetic consideration. Plant Growth Regulation. 50, (2006): 121-138.

17. Mittler, R., Abiotic stress, the field environment and stress combination; TRENDS in Plant Science. 11.1, (2006): 15-19.

18. Molla, K.A., Debnath, A.B., Ganie, S.A., Mondal, T.K., Identification and analysis of novel salt responsive candidate gene based SSRs (cgSSRs) from rice (Oryza sativa L.). BMC Plant Biology. 15, (2015):122. Doi: 10.1186/s12870-015-0498-1.

19. Mostajeran, A., Rahimi-Eichi, V., Effects of Drought Stress on Growth and Yield of Rice (Oryza sativa L.) Cultivars and Accumulation of Proline and Soluble Sugars in Sheath and Blades of Their Different Ages Leaves. American-Eurasian J. Agric. \& Environ Sci. 5.2, (2009): 264-272.

20. Muthurajan, R., Shobbar, Z.S., Jagadish, S.V.K., Bruskiewich, R., Ismail, A., Leung, H., Bennett, J., Physiological and Proteomic Responses of Rice Peduncles to Drought Stress. Mol Biotechnol. 48, (2011): 173-182.

21. Nachimuthu, V.V., Muthurajan, R., Duraialaguraja, S., Sivakami, R., Pandian, B.A., Ponniah, G., Gunasekaran, K., Swaminathan, M., Suji, K.K., Sabariappan, R., Analysis of Population Structure and Genetic Diversity in Rice Germplasm Using SSR Markers: An Initiative Towards Association Mapping of Agronomic Traits in Oryza Sativa. Rice. 8, (2015): 30. Doi: 10.1186/s12284-015-0062-5.

22. Nakashima, K., Takasaki, H., Mizoi, J., Shinozaki, K., Yamaguchi-Shinozaki, K., NAC transcription factors in plant abiotic stress responses. Biochimica et Biophysica Acta. 1819, (2012): 97-103.

23. Rabbani, M.A., Maruyama, K., Abe, H., Khan, M.A., Katsura, K., Ito, Y., Yoshiwara, K., Seki, M., Shinozaki, K., Yamaguchi-Shinozaki, K., Monitoring Expression Profiles of Rice Genes under Cold, Drought, and High-Salinity Stresses and Abscisic Acid Application Using cDNA Microarray and RNA Gel-Blot Analyses. Plant Physiol. 133, (2003): 17551767.

24. Rabello, A.R., Guimarães, C.M., Range, 1 P.H.N., da Silva, F.R., Seixas, D., de Souza, E., Brasileiro, A.C.M., Spehar, C.R., Ferreira, M.E., Mehta, Â., Identification of drought-responsive genes in roots of upland rice (Oryza sativa L). BMC Genomics. 9, (2008): 485. Doi: 10.1186/1471-2164-9-485.

25. Ruíz-Sánchez, M., Armadab, E., Munoza, Y., de Salamonec, I.E.G., Arocab, R., Ruíz-Lozano, J.M., Azcón, R., Azospirillum and arbuscular mycorrhizal colonization enhance rice growth and physiological traits under well-watered and drought conditions. Journal of Plant Physiology. 168, (2011): 1031-1037.

26. Ruíz-Sánchez, M., Aroca, R., Munoz, Y., Polon, R., Ruíz -Lozano, J.M., The arbuscular mycorrhizal symbiosis enhances the photosynthetic efficiency and the antioxidative response of rice plants subjected to drought stress. Journal of Plant Physiology. 167, (2010): 862-869.

27. Salekdeh, G.H., Siopongco, J., Wade, L.J., Ghareyazie, B., Bennett, J., Proteomic analysis of rice leaves during drought stress and recovery. Proteomics. 2, (2002): 1131-1145.

28. Sharma, P., Dubey, R.S., Drought induces oxidative stress and enhances the activities of antioxidant enzymes in growing rice seedlings. Plant Growth Regulation. 46, (2005): 209-221.

29. Shukla N, Awasthi RP, Rawat L, Kumar J Biochemical and physiological responses of rice (Oryza sativa L.) as influenced by Trichoderma harzianum under drought stress. Plant Physiology and Biochemistry. 54, (2012): 78-88.

30. Siddiqui, Z.S., Cho, J.I., Kwon, T. R., Ahn, B.O., Lee, K.S., Jeong, M.J., Ryu, T.H., Lee, S.K., Park, S.C., Park, S.H., Physiological mechanism of drought tolerance in transgenic rice plants expressing Capsicum annuum methionine sulfoxide reductase B2 (CaMsrB2) gene. Acta Physiol Plant. 36, (2014): 1143-1153.

31. Singh, C.M., Kumar, B., Mehandi, S., Chandra, K., Effect of Drought Stress in Rice: A Review on Morphological and Physiological Characteristics. Trends in Biosciences. 5(4), (2012): 261-265.

32. Tian Tian, X.J., Long, Y., Wang, J., Zhang, J.W., Wang, Y.Y., Li, W.M., Peng, Y.F., Yuan, Q.H., Pei, X.W., De novo Transcriptome Assembly of Common Wild Rice (Oryza rufipogon Griff.) and Discovery of Drought-Response Genes in Root Tissue Based on Transcriptomic Data. PLoS ONE. 10.7, (2015). Doi: 10.1371/journal.pone. 0131455.

33. Tolleter, D., Hincha, D.K., Macherel, D., A mitochondrial late embryogenesis abundant protein stabilizes model membranes in the dry state. Biochimica et Biophysica Acta. 1798, (2010): 19261933.

34. Travis, A.J., Norton, G.J., Datta, S., Sarma, R., Dasgupta, T., Savio, F.L., Macaulay, M., Hedley, P.E., McNally, K.L., Sumon, M.H., Islam, M.R., Price, A.H., Assessing the genetic diversity of rice originating from Bangladesh, Assam and West Bengal. Rice. 8, (2015):35. Doi: 10.1186/s12284015-0068-z.

35. Trenberth, K.E., Changes in precipitation with climate change. Climate Research 47.1, (2011): 123 138.

36. Tuberosa, R., Salvi, S., Genomics-based approaches to improve drought tolerance of crops. TRENDS in Plant Science. 118, (2006): 405-412. 
37. Wang, D., Pan, Y., Zhao, X., Zhu, L., Fu, B., Li, Z., Genome-wide temporal-spatial gene expression profiling of drought responsiveness in rice. BMC Genomics. 12, (2011):149. Doi: 10.1186/1471-216412-149.

38. Xia, H., Zheng, X., Chen, L., Gao, H., Yang, H., Long, P., Rong, J., Lu, B., Li, J., Luo, L., Genetic Differentiation Revealed by Selective Loci of Drought-Responding EST-SSRs between Upland and Lowland Rice in China. PLoS ONE. 9(10), (2014). Doi: 10.1371/journal.pone.0106352.

39. Xiong, J.H., Fu, B.Y., Xu, H.X., Li, Y.S., Proteomic analysis of PEG-simulated drought stress responsive proteins of rice leaves using a pyramiding rice line at the seedling stage. Botanical Studies. 51, (2010): 137145.
40. Xu, J., Yuan, Y., Xu, Y., Zhang, G., Guo, X., Wu, F., Wang, Q., Rong, T., Pan, G., Cao, M., Tang, Q., Gao, S., Liu, Y., Wang, J., Lan, H., Lu, Y., Identification of candidate genes for drought tolerance by whole-genome resequencing in maize. BMC Plant Biology. 14, (2014):83. Doi: 10.1186/1471-2229-14-83.

41. Zhang, Z.F., Li, Y.Y., Xiao, B.Z., Comparative transcriptome analysis highlights the crucial roles of photosynthetic system in drought stress adaptation in upland rice. Scientific Reports 6, (2016). Doi: 10.1038/srep19349.

\section{Cite this article as:}

Shamsun Nahar, Jyotirmay Kalita, Lingaraj Sahoo, Bhaben Tanti. Morphophysiological and molecular effects of drought stress in rice. Annals of Plant Sciences 5.9 (2016): 1409-1416.

DOI: http://dx.doi.org/10.21746/aps.2016.09.001

Source of support: UGC - Maulana Azad National Fellowship, India. Conflict of interest: None Declared 